\title{
DETERMINING THE SOLUBILITY AND CRYSTAL FORM OF CLENBUTEROL IN THIN FILMS OF EUDRAGIT NE30D
}

\author{
Achim Göpferich and Geoffrey Lee ${ }^{1}$ \\ Institute for Pharmaceutical Technology and Biopharmaceutics, \\ Heidelberg University, Germany.
}

\section{ABSTRACT}

The solubility of the drug clenbuterol in thin films of surfactant-free Eudragit NE30D has been measured. Light microscopy and differential scanning calorimetry were supplemented by a technique based on measurement of the rate of drug release from the films. The clenbuterol crystals had the form of a fractal, as could be shown by a computer simulation of diffusion-controlled aggregation.

\section{INTRODUCTION}

When preparing polymeric, sustained-release carriers for a drug, it is useful to know the solubility of the drug in the polymer. The release kinetics depend inter alia on whether the drug exists in the dissolved or the

\footnotetext{
1Correspondence: Prof. Geoffrey Lee, Institut für Pharmazeutische Technologie und Biopharmazie, Im Neuenheimer Feld 366, 6900 Heidelberg, Germany.
} 
suspended condition (1). We wished to determine the solubility of the drug clenbuterol in thin films of the polacrylate Eudragit NE30D. Both polarising light microscopy and differential scanning calorimetry (DSC) offer themselves as methods. With the former, the solubility is estimated by identifying the drug concentration at which crystals are first observed to form within the polymer. With DSC, the first appearance of a drug melting peak identifies the presence of solid drug (2), and hence the limit of solubility. We supplement these methods with two others, based on measurement of the rate of drug release from the thin films. If such a film contains only dissolved drug, then the release process can be described by a simple, diffusional model (3a). If, however, the film also contains suspended drug, the rate of drug release will be altered (1). We exploit this difference in release rate to estimate drug solublity within the film. With the first technique, a non-steady state model for the presence of dissolved drug is fitted to experimental drug release data obtained for films having a wide range of drug loading. The shape of the functional relationship between fitted diffusivity and drug loading can then be used to estimate at which drug loading suspended drug first appears within the films. With the second technique, this model is extended to account for the presence of suspended drug. This is readily achieved by using a numerical method, which has the added advantage that realistic, non-sink boundary conditions can be recognized. By fitting this model to experimental drug release results, a direct calculation of the drug solubility within the film should be possible. In the present paper we contrast the results obtained with these methods with those obtained using microscopy and DSC. Additionally, we relate some observations of the crystal form of clenbuterol within the polymer film. 


\section{MATERIALS AND METHODS}

Preparation of Drug-Loaded Thin Films: Frieze dried Eudragit NE30D (Röhm, Weiterstadt, Germany) was first refluxed for $90 \mathrm{~h}$ in water to remove its indiginous surfactant. This procedure is necessary to ensure an isotropic system (4). Drug-loaded films of $c a .50 \mu \mathrm{m}$ thickness were then prepared from this purified polymer by solvent evaporation, as described before (5). The basic drug clenbuterol (Boehringer Ingelheim, Ingelheim, Germany) was used ( $\mathrm{pKa}=8.85)$ :

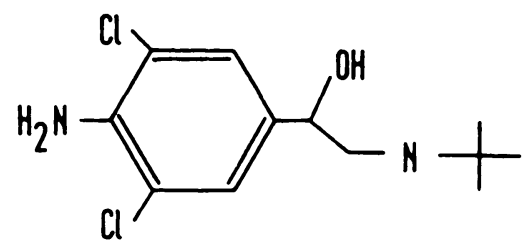

Thin films having drug loadings in the range $0-25 \% \mathrm{w} / \mathrm{w}$ were prepared, and the thickness of each determined using an Elcometer (Elcometer Instruments, Manchester, England).

Estimation of Solubility from Drug Release Rate: The rate of release of clenbuterol from each thin film into a $\mathrm{pH} 8$ phosphate buffer solution was determined using a horizontal diffusion cell, as described before (6). Each result was expressed as a release profile of $m_{a}(t) / m_{o}$ versus $t$, where $m_{a}(t)$ is the mass of drug released after time $t$, and $m_{o}$ is the original mass of drug in the film at $t=0$. Two diffusional models were then used to estimate the solubility of the drug in the films. The first model is for drug release from a film containing only dissolved drug. The linear form of the diffusion equation is solved using a numerical, finite-difference technique, as 
a)

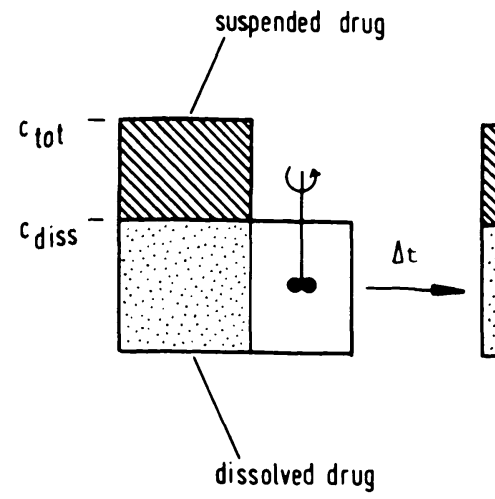

b)

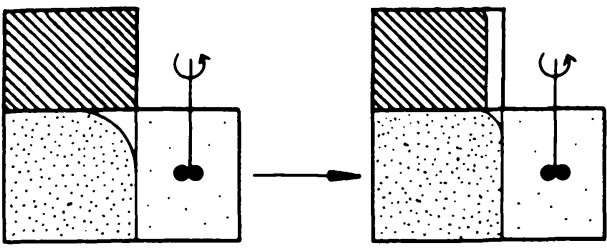

c)

\begin{abstract}
FIGURE 1
Model for drug release from a thin film containing dissolved and suspended drug.
\end{abstract}

described before (6). Fitting this model to the experimental release profiles using an improved simplex method (7) yielded the best value for drug diffusivity, D. This was then plotted against drug loading to estimate the drug solubility. The second model is an extension of the first, and is illustrated in Fig.1. We define the total concentration of drug within the film, $c_{\text {tot }}(x, t)$, to consist of the concentrations of both dissolved, $c_{d i s s}(x, t)$, and suspended, $c_{\text {susp }}(x, t)$, drug (Fig. 1a). The numerical solution to the diffusion equation must be modified to account for the presence of suspended drug. Thus, at $t=0$, the value of $c_{\text {diss }}(x, t)$ is equal to the solubility of the drug in the film, $c_{\mathbf{s}}$, and decreases with time as a result of diffusion out of the film (Fig. 1b). After each time step of the numerical calculation, $\Delta t$, the value of $c_{\text {diss }}(x, t)$ is increased spontaneously to $c_{s}$ at those points where suspended drug is still present (Fig. 1c). The concentration of suspended drug remaining at these points is accordingly reduced, eventually falling to zero 


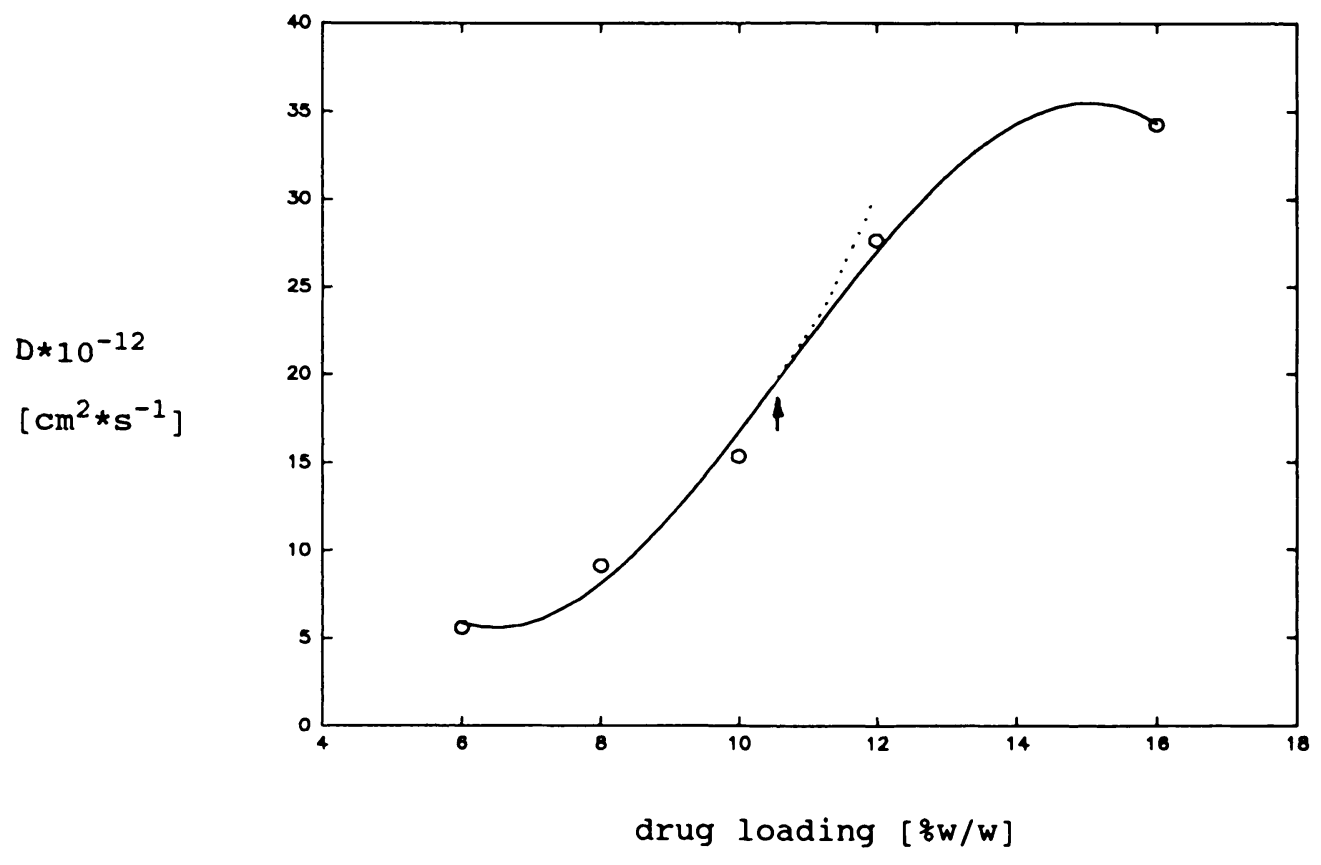

FIGURE 2

Clenbuterol diffusivity (D) versus drug loading of thin films of Eudragit NE30D. Arrow: estimated solubility of drug.

and leaving only dissolved drug present. The solution to the model yields both theoretical concentration-distance profiles of drug within the film, and theoretical drug release profiles of $\mathrm{m}_{\mathrm{a}}(\mathrm{t}) / \mathrm{m}_{\mathrm{O}}$ versus $\mathrm{t}$. The latter were then fitted to each experimental release profile as before. In this case, however, the best value for drug solubility within the film is obtained directly.

Microscopical Examination of Thin Films: The thin films were examined under a polarising light microscope with hot stage (Zeiss Standard), and photographs taken with a Nikon C35 camera (Nikon, Japan). 


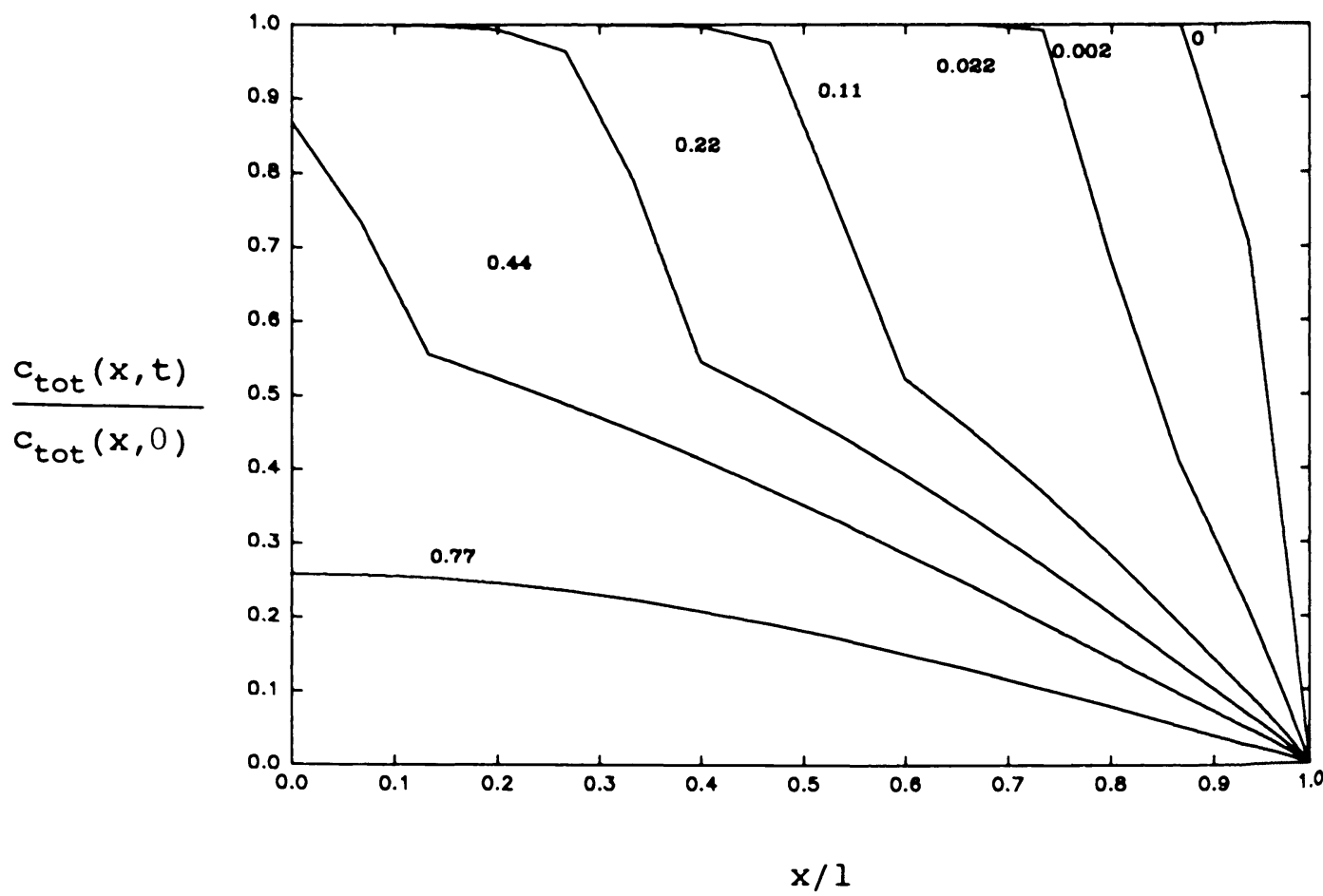

FIGURE 3a

Simulated drug concentration profiles within thin film for different values of $\mathrm{Dt} / 1^{2}(40 \% \mathrm{w} / \mathrm{w}$ suspended drug).

Thermal Analysis of Thin Films: The thermal behaviour of the thin films was examined using differential scanning calorimetry (DSC) with a Mettler Model TC10A (Greifensee, Switzerland). A heating/cooling rate of $10 \mathrm{~K} / \mathrm{min}$ was used over the temperature range $28(1)-405 \mathrm{~K}$.

\section{RESULTS AND DISCUSSION}

It is known that drug diffusivity within an isotropic film can be calculated by fitting the model for dissolved drug to experimental release 
$\frac{m_{a c c}(t)}{c_{d}(x, 0) \star A \star 1}$

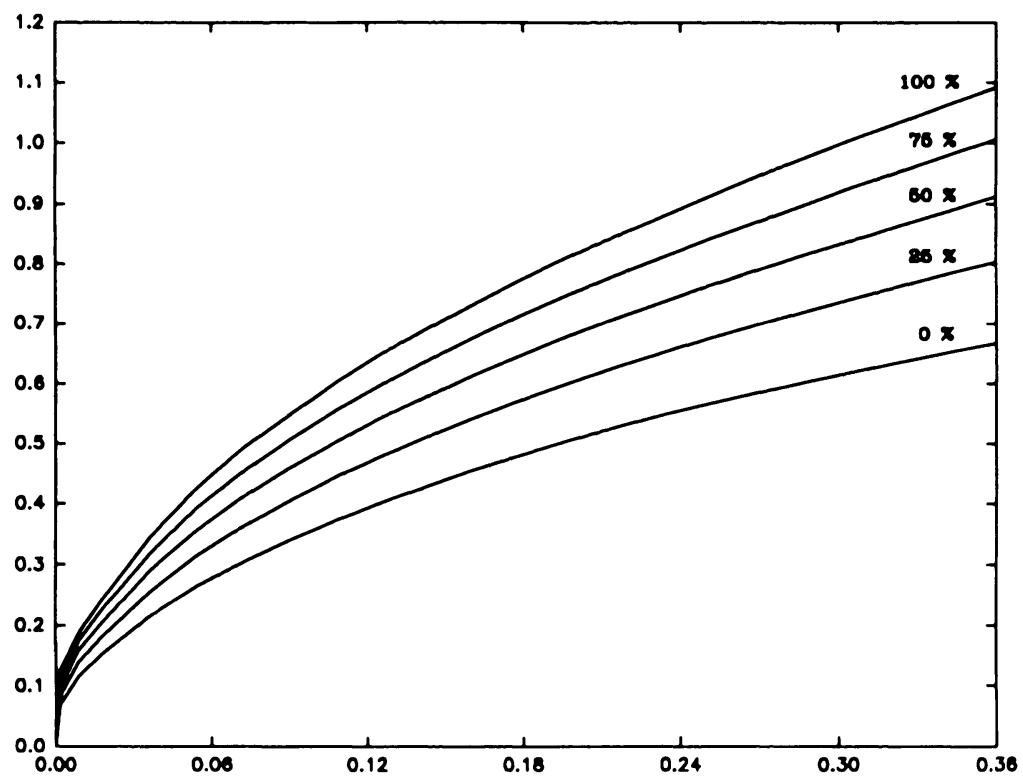

$$
D * t / 1^{2}
$$

\section{FIGURE 3b}

Simulated drug release profiles for increasing amount of suspended drug in thin film.

profiles (6). The fitted diffusivities obtained for the present system are dependent on the drug loading (Fig. 2), as is found for many systems (3b). Using the relation: $\mathrm{D}(\mathrm{c})=\mathrm{D}_{\mathrm{O}} \mathrm{e}^{\mathrm{kc}}$, the results at low drug loadings (up to the arrow on Fig. 2) yield the values $D_{0}=7.58 \times 10^{-12} \mathrm{~cm}^{2} / \mathrm{s}$ and $\mathrm{k}=0.16$ $\mathrm{cm}^{3} / \mu \mathrm{g}$. At high drug loadings (above the arrow on Fig. 2), the experimental coordinates deviate from this exponential relation. This effect can be ascribed to the formation of drug crystals within the film at high drug loadings. In the presence of drug crystals, the concentration of dissolved 


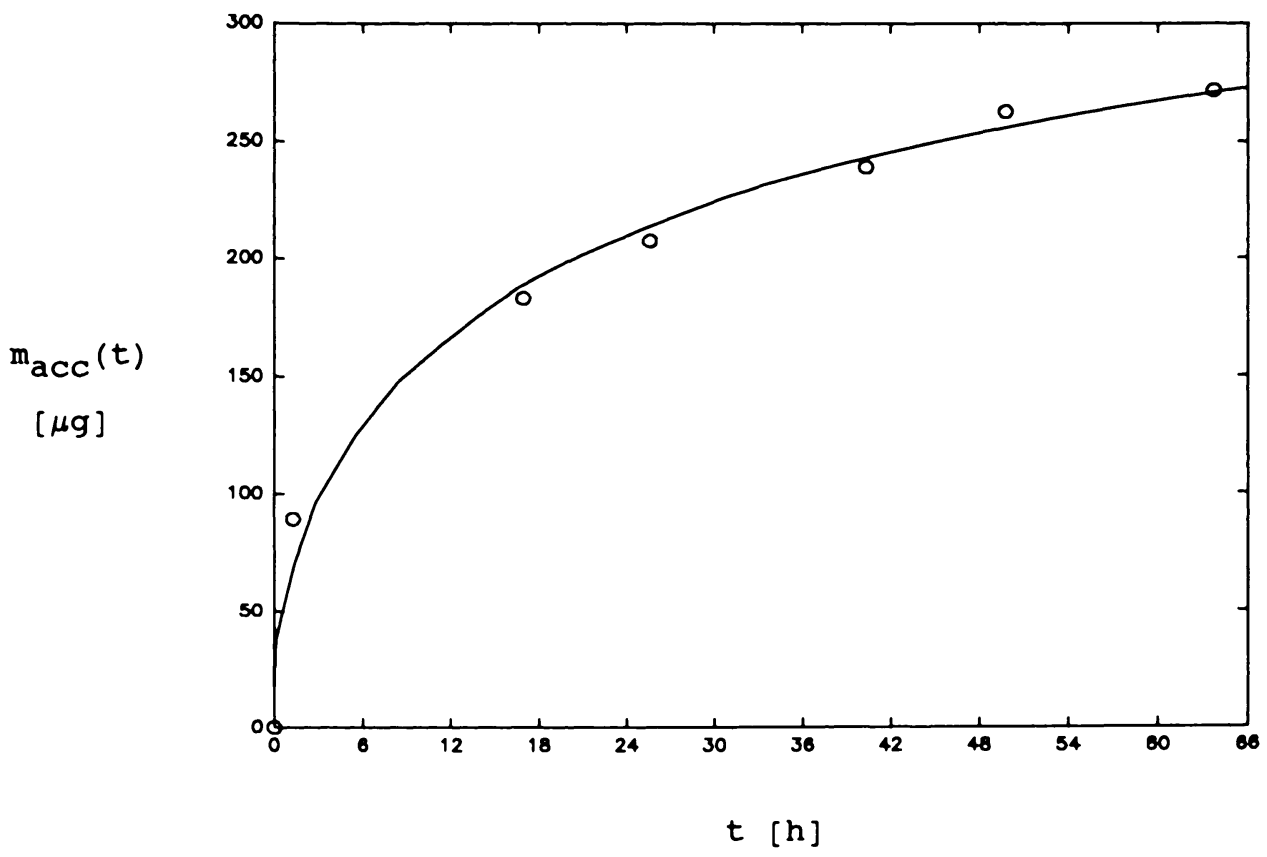

\section{FIGURE 4}

Release of clenbuterol from thin film of Eudragit NE30D. Coordinates: experimental data. Curve: theoretical solution of best fit.

drug within the film will be overestimated, and the fit of the model to the experimental data will yield an underestimated diffusivity. In this case, the point of departure of the experimental results from the exponential curve should correspond to the appearance of crystallized drug in the film. This occurs in Fig. 2 at a value of $10.6 \% \mathrm{w} / \mathrm{w}$ clenbuterol, which we take to be the solubility of the drug in the polymer.

The second model recognizes the influence of the presence of suspended drug on the release process, as can be appreciated from the theoretical concentration-distance profiles within a film shown in Fig. 3a. 
The discontinuity in each profile represents the moving boundary existing between the inner region of the film containing both dissolved and suspended drug and the outer region, where only dissolved drug remains. The profiles are curved, as a result of the non-steady state nature of the model, in contrast to steady state models (8). The theoretical release profiles in Fig. 3b illustrate how an increasing concentration of suspended drug within the film produces increased rates of drug release at constant diffusivity. This effect can be exploited to determine drug solubility within the film. Thus, Fig. 4 shows a typical experimental release profile (coordinates) together with the best fit of the model to these coordinates (curve). From this fit it was possible to determine not only the diffusivity but also the solubility. The results are compiled in the Table for two drug loadings. The values for $\mathrm{D}$ are somewhat higher than those obtained with the first model for only dissolved drug (cf. Fig. 2). The presence of suspended drug is recognized with the second model, thereby avoiding the aforementioned overestimation of the concentration of dissolved drug within the film. Of interest here is the value obtained for the solubility of clenbuterol, $\mathrm{ca} .13 .5 \% \mathrm{w} / \mathrm{w}$.

\section{TABLE}

Results of fit of theoretical model to experimental release profiles.

\begin{tabular}{|c|c|c|}
\hline $\begin{array}{l}\text { Drug loading } \mathrm{n} \\
{[\% \mathrm{w} / \mathrm{w}]}\end{array}$ & $\begin{array}{l}\mathrm{D} \times 10^{-11} \\
{\left[\mathrm{~cm}^{2} / \mathrm{s}\right]}\end{array}$ & $\left.c_{\delta} / \% \mathrm{w} / \mathrm{w}\right]$ \\
\hline $\begin{array}{l}16 \\
20\end{array}$ & $\begin{array}{l}4.18 \pm 0.899 \\
2.68 \pm 0.114\end{array}$ & $\begin{array}{l}13.5 \pm 0.2 \\
13.8 \pm 0.3\end{array}$ \\
\hline
\end{tabular}




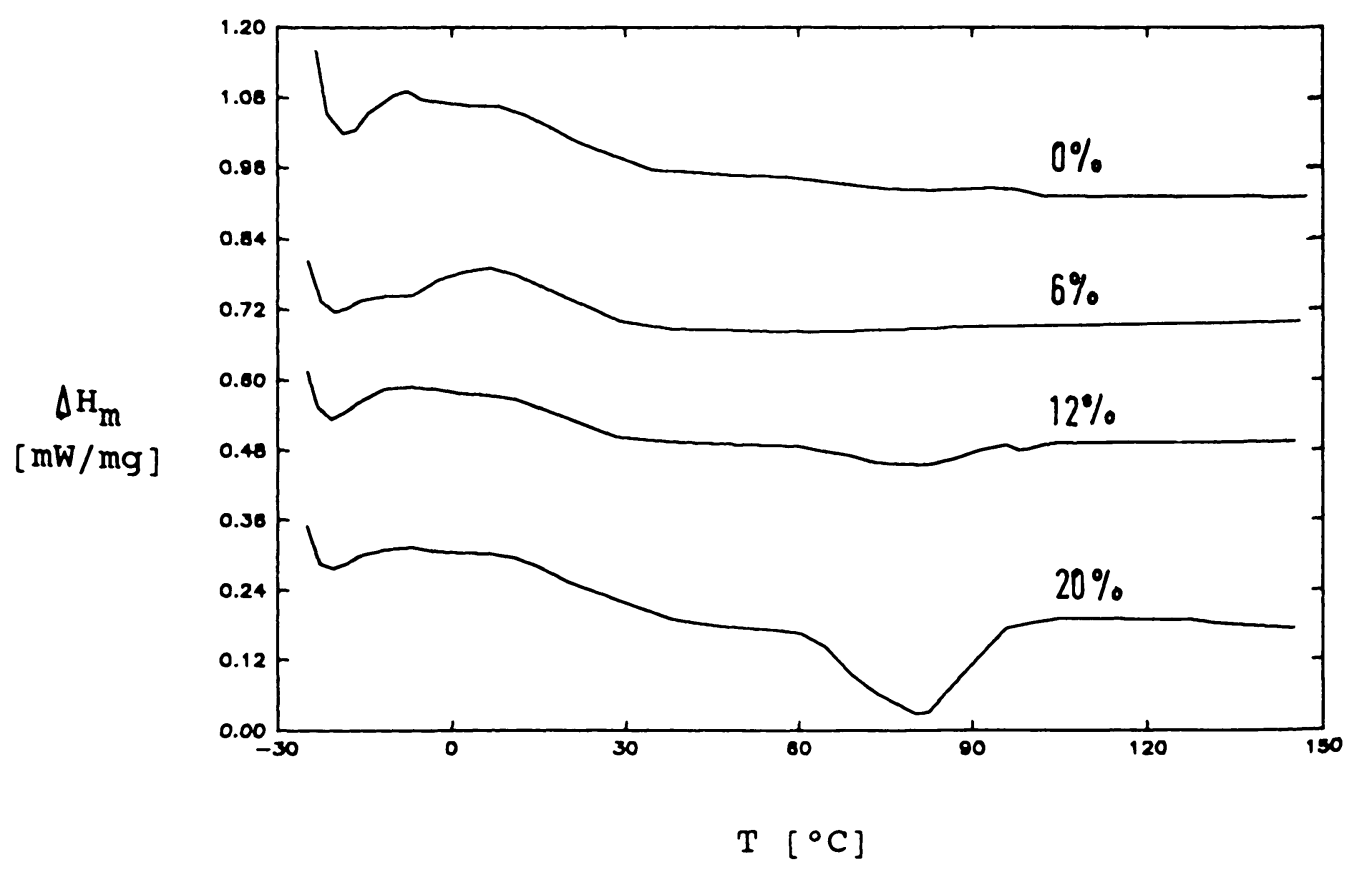

\section{FIGURE 5}

DSC-thermograms for clenbuterol-loaded thin films of Eudragit NE30D.

This value is higher than that obtained from the first model. It is also higher than that suggested from the microscopy, where it was possible to observe the presence of crystals at clenbuterol loadings as low as ca. $10 \%$ $\mathrm{w} / \mathrm{w}$. These melted at $c a .85^{\circ} \mathrm{C}$, a temperature substantially lower than the melting point of the pure drug and indicating some interaction of the drug with the polymer. The corresponding DSC-thermograms (Fig. 5) first show a recognizable drug melting peak at a drug loading of $c a .12 \% \mathrm{w} / \mathrm{w}$. The microscopical method is, therefore, evidently more sensitive than the DSC method for estimating drug solubility in such systems. The higher value 


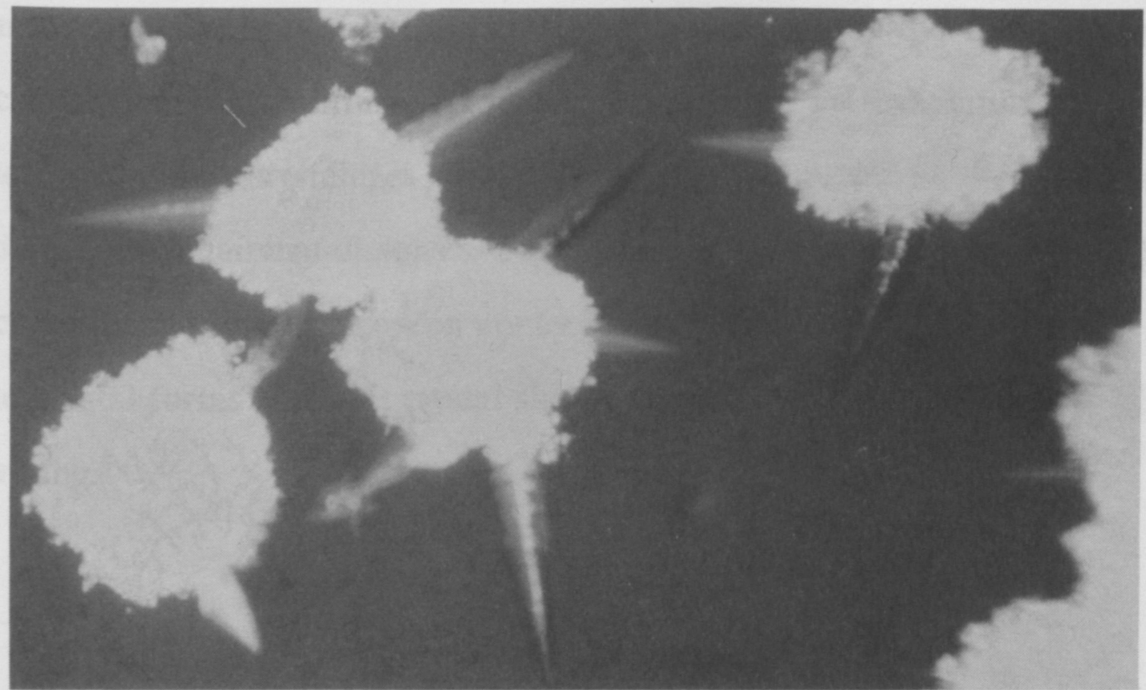

FIGURE 6a

Individual drug crystals present in thin film. (

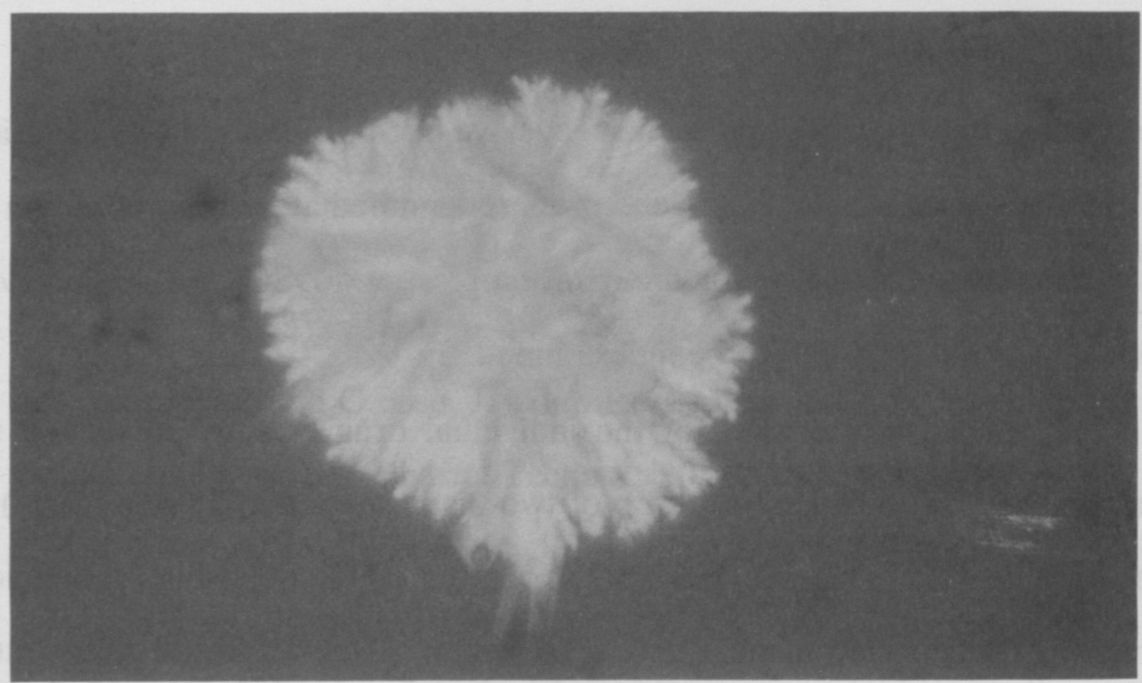

FIGURE 6b

Detail of central aggregate of drug crystal in thin film. Scale as above. 

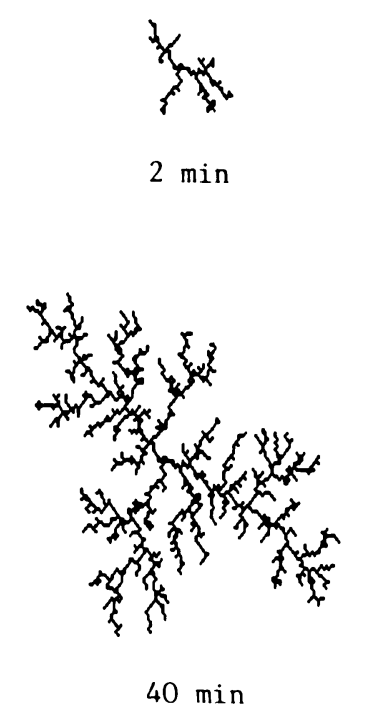

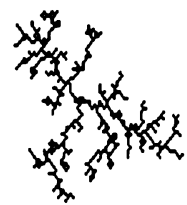

$10 \mathrm{~min}$

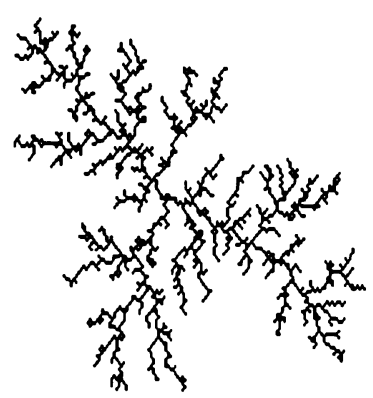

$120 \mathrm{~min}$

\section{FIGURE 7}

Simulation of crystal growth by diffusioncontrolled aggregation.

found from the release experiments may have been caused by oversaturation of the films with drug. They were prepared immediately before use in an experiment, whereas those for the microscopical and DSC experiments were stored for some days before being examined.

A careful examination of the individual drug crystals present in the films reveals that some are built up of two distinct crystal forms (Fig. 6a). A central crystal is present, which is surrounded by feathery-like projections spreading out into the surrounding polymer. The shape of the central aggregate (Fig. 6b) indicates that it is a fractal. It can indeed be obtained almost exactly by a simple computer simulation of diffusion-controlled aggregation within an isotropic medium (Fig. 7). For this simulation it was 
assumed that the molecules are initially dissolved within the film, through which they can freely diffuse. At single points within the oversaturated film, some of the drug crystallizes to form a seed. As a result of their thermal motion, the remaining dissolved drug molecules undergo a randon walk, which stops by crystallization on contact with the seed (9). The similarity of these crystal forms with the crystal shapes observed within the polymer film is striking.

\section{ACKNOWLEDGEMENTS}

We are thankful to Boehringer Ingelheim for its support of this project.

\section{REFERENCES}

(1) L. Fan and S. Singh, "Controlled Release, A Quantitative Treatment", Springer Verlag, Heidelberg 1989, p. 25.

(2) R. Bodmeier and O. Paeratakal, Pharm. Res., 6, 725 (1989).

(3) J. Crank, "The Mathematics of Diffusion", Oxford University Press, London, 1975. a) p. 56; b) p. 105.

(4) A. Göpferich and G. Lee, J. Cont. Rel., submitted.

(5) B. Zierenberg, Acta Pharm. Technol., 30, 17 (1985).

(6) G. Göpferich and G. Lee, Int. J. Pharm., 71, 245 (1991).

(7) J. Nelder and R. Mead, Computer J., 7, 308 (1967).

(8) T. Higuchi, J. Pharm. Sci., 50, 874 (1961).

(9) T. Whitten and L. Sander, Phys. Rev. Let., 47, 1400 (1981). 